\title{
CTS DESDE LA PERSPECTIVA DE LA EDUCACIÓN TECNOLÓGICA
}

\section{Tomás Buch (*)}

SÍNTESIS: En sus aspectos educativos, el área general de la tecnología aún no ha logrado un lugar universalmente reconocido en los sistemas educativos de los diferentes países, en los que coexisten concepciones diferenciadas acerca de sus objetivos y de sus contenidos. El tema ha sido abordado desde dos ángulos diferentes: la corriente Ciencia, Tecnología y Sociedad (CTS), y lo que, con diversas acepciones del término, se denomina educación tecnológica. En el intento de lograr una estructuración del área, se ha considerado la posibilidad de enfocar la educación tecnológica desde el ángulo CTS. En este trabajo se explora la posibilidad opuesta: la de estudiar las relaciones entre la Ciencia, la Tecnología y la Sociedad desde el ángulo de cierta forma de abordar la educación tecnológica.

SÍNTESE: Em seus aspectos educativos, a área geral da tecnologia ainda não logrou um lugar universalmente reconhecido nos sistemas educacionais dos diferentes países, onde coexistem conceitos diferenciados a respeito de seus objetivos e de seus conteúdos. O tema tem sido abordado a partir de dois ângulos diferentes: a corrente Ciência, Tecnologia e Sociedade (CTS), e aquilo que, com diversas acepções do termo, denomina-se educação tecnológica. Com o objetivo de obter uma estruturação da área, considerou-se a possibilidade de enfocar a educação tecnológica a partir do ângulo CTS. Neste trabalho se explora a possibilidade oposta: a de estudar as relações entre a Ciência, a Tecnologia e a Sociedade a partir da perspectiva que proporciona a educação tecnológica.

\section{INTRODUCCIÓN}

Hace ya unos cuantos años que en muchos países se realizan intentos de lograr que en las escuelas se difundan conocimientos sobre tecnología. Estos esfuerzos se ponen de manifiesto en los planes de

(*) Consultor de la gerencia general del INVAP S.E. y del Grupo Argentino de Educación Tecnológica (GAET), Argentina. 
estudio de todos los niveles de la educación, desde el inicial (jardín de infantes) hasta el universitario, al margen de los conocimientos profesionales que imparten las escuelas técnicas y las facultades de ingeniería. Como es obvio, en cada uno de estos niveles los esfuerzos tienen características muy diferentes, pero siempre con el objetivo de lograr una comprensión conceptual, actitudinal y procedimental de la artificialidad en general, y de los aspectos históricos y contemporáneos de la tecnología, procurando una articulación entre las habilidades técnicas del sujeto, su conocimiento del mundo artificial en el que debe moverse, sus capacidades cognitivo-afectivas, sus valores y su actitud ante la naturaleza ${ }^{1}$.

La finalidad de esos estudios es la de que los ciudadanos puedan desempeñarse mejor en una sociedad altamente tecnificada, a la vez que entiendan sus limitaciones y sus peligros. Esto incluye la capacitación para poder participar en la toma de decisiones en lo que respecta a los temas tecnológicos polémicos con un conocimiento de causa suficiente, lo que no siempre es fácil, dada la complejidad de los factores que inciden sobre tales decisiones.

Llama la atención el hecho de que la necesidad de que todos los ciudadanos tengan algunos conocimientos generales acerca de las tecnologías más significativas aún no haya obtenido una aceptación unánime, como lo pone de manifiesto un reciente trabajo publicado en este mismo medio (Gordillo y González Galbarte, 2002), que aboga calurosamente por la educación tecnológica (ET) y propone el enfoque CTS para vehicular su aceptación.

Esto evidencia una primera dificultad para lograr el objetivo, que proviene de las diversas acepciones en las que el término ET ha sido empleado. El más general parece ser, sencillamente, la introducción de los conocimientos de y sobre la tecnología, tal como los distingue Gilbert (Gilbert, 1992). Pero la diversidad de enfoques sobre la ET es bastante notable (Acevedo, sf). Más adelante describiremos en detaIle el sentido en el que proponemos usar ese término, y mostraremos que la corriente que representa es diferente a la que se suele designar con la sigla CTS en algunos aspectos esenciales, pero en la que puede hallarse una complementación entre ambas maneras de abordar, a

1 Agradezco sus valiosos comentarios a los miembros del GAET Abel Rodríguez de Fraga, Carlos Marpegán y César Linietzky. 
nivel del sistema educativo, el necesario acercamiento entre la sociedad y la tecnología de la cual es cada vez más dependiente, pero a la que aún se resiste a conocer en toda su complejidad. Dicha complementación puede basarse en el hecho de que la ET -en la versión que proponemos en éste y en otros trabajos- se adapta singularmente a la introducción de la tecnología en toda su amplitud conceptual desde los primeros años de la escuela básica, aunque no se limita a ellos (Rodríguez de Fraga, 1999).

En lo que se entiende por educación tecnológica hay varios enfoques, que incluso difieren en su finalidad y en su metodología (Gilbert, 1995). En efecto, para algunos (Argüelles, 1999), la ET es sobre todo una preparación para la vida laboral, mientras que para otros (Rodríguez de Fraga, 1996) es una introducción al modo de pensar y de actuar del tecnólogo, o, dicho con más propiedad, del ser humano en tanto actúa como tecnólogo, empleando su propio cuerpo o las herramientas cada vez más complejas que lo complementan, para modificar su ambiente, modificándose a sí mismo en el proceso.

Este último concepto abarca un amplio espectro de temas, desde las consideraciones de raigambre psicológica y antropológica sobre la relación, mediada por los instrumentos entre el hombre y el mundo exterior (Verillion, 2000), hasta el enfoque sistémico de las estructuras tecnológicas y de los sistemas técnicos de las diversas civilizaciones (Buch, 1999). Esta secuencia se presta para ser presentada a los alumnos de los diferentes niveles y para ser trabajada por ellos en la medida de su maduración psicocognitiva, desde los primeros niveles educativos hasta la adolescencia.

En cada nivel educativo, a su vez, cada uno de estos temas tiene su reflejo pedagógico y didáctico, y en cada uno de los niveles la discusión está lejos de haber conducido a consensos. En efecto, la interacción de cada una de estas orientaciones con la práctica experimental en el aula es bastante variada, lo que refleja los orígenes históricos de cada una de ellas. Mientras que CTS tiene un fuerte componente histórico y se basa en las llamadas humanidades, lo que se suele entender por ET tiene más apoyo en las técnicas duras, lo que ha podido alimentar una posición equívoca y la concepción prejuiciosa -con cierta tendencia a la tecnofobia- de la tecnología como algo deshumanizado u opuesto al humanismo o aún a la propia sociedad. 


\section{CIENCIA, TECNOLOGÍA Y SOCIEDAD}

Los contenidos que propone la corriente CTS (González García, López Cerezo y Luján López, 1996) más bien están dirigidos a la educación secundaria no técnica, y, por lo general, son medianamente críticos de muchos aspectos del estilo de desarrollo tecnológico característico del capitalismo tardío. El interés por los estudios CTS tiene su origen histórico en el reconocimiento de los peligros a los que la evolución tecnológica actual expone a la humanidad (Mumford, 1970; Winner, 1979, 1987), y no por acaso el primer término del trinomio que nos presenta es la ciencia. Desde su aparición como instrumento esencial en el desarrollo de nuevas tecnologías, la ciencia ha jugado un papel creciente en el imaginario social, pero no sólo como conocimiento puro de la naturaleza sino como fuente y origen de los logros tecnológicos modernos, hasta el punto de confundirse, en algunos casos, con la tecnología misma. Ese creciente impacto de la ciencia sobre la tecnología, cuyo comienzo se puede ubicar en la segunda mitad del siglo XIX pero que no ha dejado de acelerarse desde entonces, ha conducido a la imposición de acepciones falsamente unívocas para términos que admiten varias definiciones. Así, se llega hasta el extremo de reservar el término «tecnología» para aquellas tecnologías basadas más o menos en las aplicaciones del conocimiento científico (Bunge, 1996). Tal confusión ha tenido graves consecuencias en todos los niveles del ámbito educativo, pues al confundir la tecnología con la mera aplicación de las ciencias que le sirven de fundamento se falsea el proceso habitual de la creación tecnológica hasta hacerlo irreconocible (Cares, 1999).

En cuanto a la visión de su impacto sobre la sociedad, cabe reconocer que, desde mediados del siglo xx, se depositaron grandes esperanzas en la potencialidad de las diversas tecnologías que se iban desarrollando con creciente eficacia a partir de la ciencia, con la convicción de que proporcionarían a la humanidad entera un futuro venturoso. Esta esperanza, basada en un optimismo progresista bastante ingenuo y de base fundamentalmente ideológica, tuvo como coadyuvantes ciertas corrientes de pensamiento para las que la tecnología era una especie de entelequia en la que se afirmaba, aun lamentándolo, cierto nivel de autonomía (Winner, 1979). Al mismo tiempo, en la opinión pública predominaba la convicción de que la tecnología iba a resolver los problemas sociales, como los fenómenos del subdesarrollo, el crecimiento desordenado de las ciudades en el tercer mundo, el abuso de los recursos naturales y la creciente contaminación ambiental, cuyo avance se percibía con claridad. Sin embargo, en la medida en que poco a poco 
estos efectos nocivos de la civilización mundializada se percibían con mayor nitidez, y en la medida en que se iban agravando los problemas en vez de resolverse como esperaban los optimistas, el estado de ánimo de los observadores fue cambiando, y el optimismo tecnofílico fue transformándose en un pesimismo y en una tecnofobia crecientes ${ }^{2}$. Es en el ámbito de este pesimismo en el que se origina la corriente CTS, que destaca el carácter social de todas las decisiones tecnológicas y trata de analizar su génesis y sus consecuencias. Lejos de ser una fuerza autónoma, la tecnología es la expresión de las relaciones de propiedad y de poder en la sociedad. Es más, deberíamos decir en las sociedades, ya que no es lo mismo generar e introducir una tecnología determinada en un país desarrollado que en uno del tercer mundo (Dagnino y Thomas, 2000). Se reeditan así, en las diversas vertientes de la corriente CTS, orientaciones que van desde un determinismo social o económico hasta otro tecnológico, pasando por opciones neutralistas, cada una en diversas versiones más duras o más blandas (Dagnino, sf).

Las críticas al modelo de desarrollo predominante se han hecho cada vez más frecuentes, tanto en los ámbitos CTS y de los estudios sociales de la Ciencia y la Tecnología, como en los medios y ambientes de debate más generales, sin excluir los medios de difusión masiva. En ciertos casos, los llamados a una mayor sensatez en las opciones de desarrollo se han hecho casi desesperados, ante la falta de respuesta real y concreta del sistema económico globalizado por los riesgos evidentes y por sus graves consecuencias visibles ya claramente (Morin, 2002). El concepto de «desarrollo sustentable» se ha convertido en tema de importantes conferencias internacionales, cuyas resoluciones pocas veces conducen a cambios perceptibles en el comportamiento de los factores de poder, porque afectan intereses económicos demasiado poderosos (Universidad Libre de Bruselas, 2002).

En este punto quisiéramos hacer una observación importante: las críticas a las tecnologías contemporáneas y sus efectos se han hecho oír más que nada en los foros internacionales y en los países desarrollados, que, así como lideran todos los adelantos, también lo hacen con los estudios que expresan estas críticas. Por tal motivo, se corre el peligro de que la evaluación del impacto social de la CyT sea efectuado

2 Por ejemplo, en la simple enumeración de los contenidos de la signatura de CTS en la Educación Secundaria Obligatoria (ESO) de España (1999) hay implícitas ciertas tomas de posición, p. ej. el punto 4.5 se llama «Ética nuclear y ética medioambiental», aparentemente presuponiendo un contraste entre ambas. 
exclusivamente desde la óptica de dichos países, cuando se puede pensar que una visión centrada en las necesidades y oportunidades de los países de mediano nivel de desarrollo sea bastante diferente ${ }^{3}$. Esto no debe interpretarse en el sentido de que los países menos adelantados tendrían que aceptar tipos de desarrollo que no son sustentables en otras latitudes, con la excusa de que su pobreza los obliga a aceptar lo inaceptable; sin embargo, los estudios de impacto social, como los que se efectúan en la órbita CTS, han de tener en cuenta de formas más explícitas las diferencias entre el impacto de la ciencia, y, sobre todo, de las diversas tecnologías sobre los países periféricos, y la que tienen en los países centrales.

Asimismo, al analizar la manera en que se vincula la ciencia con la tecnología, y al estudiar cómo se adoptan y se adaptan las diversas tecnologías en los países periféricos, muchas veces se han aceptado, prima facie, los modelos aplicables en los países centrales, con lo cual la constatación de lo reducido del impacto que la investigación científica local ha tenido sobre el desarrollo económico de los países periféricos sólo ha conducido a una sensación de incomprensión y no a un mejoramiento de su inserción en las actividades productivas (Buch, 2002).

\section{LA EDUCACIÓN TECNOLÓGICA}

Al definir la ET vamos a empezar por reiterar que dicho término se aplica a cosas muy diferentes según los países. En ciertos casos, la ET no se diferencia de la educación técnica (Argüelles, 1999). La educación técnica es una modalidad tradicional de la educación secundaria que ha tendido a formar técnicos, es decir, a personas con formación profesional en el nivel secundario, que disponen de conocimientos y de experiencias que los habilitan para una actividad laboral especializada de asistencia y de ayuda a los niveles profesionales de formación universitaria. La distinción entre esta educación técnica y lo que nosotros llamamos ET adquiere especial importancia porque en varios países se están realizando reformas educativas que tienden a hacer más poliva-

3 Dos ejemplos concretos de esto se encuentran en dos tecnologías que son rechazadas o cuestionadas por muchos críticos de las tecnologías predominantes: la energía nuclear y los cultivos transgénicos. En efecto, por diversos motivos, ambas tecnologías tienen hoy en la Argentina un importante desarrollo. El ataque tecnofóbico contra ellas tiende a privar al país de importantes fuentes de ingresos, mientras que no afecta mucho a las industrias respectivas en su conjunto mundial. 
lentes a los egresados del ciclo secundario, ante la idea -cuya veracidad y pertinencia no analizaremos aquí- de que un trabajador menos especializado será más adaptable a los rápidos cambios tecnológicos, y, por lo tanto, tendrá mejores oportunidades laborales que un técnico tradicional de nivel secundario. Esta tendencia, promocionada a partir de ciertos comentarios emanados del Banco Mundial ${ }^{4}$, ha tenido diversos niveles de aceptación y de realización en diversos países, y ha sembrado el desconcierto entre alumnos y docentes de esa orientación pedagógica en todas partes.

En otros ámbitos, ET es una actividad que se concentra en los niveles más elementales de la educación, en cuyos alumnos trata de desarrollar conocimientos, procedimientos y actitudes en relación con la tecnología en su acepción más general. Es en esos niveles en los que la ET toca más de cerca los conceptos antropológicos y las actividades operacionales, logrando que los alumnos más pequeños aborden problemas tecnológicos con los medios técnicos a su disposición, al margen de las consideraciones sociológicas más aplicables a las tecnologías contemporáneas (Rodríguez de Fraga, 1994; 2002; Gennuso, 2000).

El concepto de ET que manejamos en estas líneas se conjuga con el de alfabetización tecnológica, que parte de la idea de que, cualquiera que sea su actividad laboral, un ciudadano del mundo actual no puede prescindir de una formación general en tecnología (la que simultáneamente se intenta definir como área del conocimiento), ya que el mundo en que vive es, cada vez en mayor medida, un mundo artificial, es decir, un mundo creado por la tecnología en la acepción más amplia de ese término. El uso de la expresión alfabetización tecnológica se deriva del concepto de alfabetización científica acuñado hace unos años para describir la necesidad imperiosa de que la gente disponga de conocimientos científicos para poder orientarse en el mundo actual (Fourez,

4 El Documento de política del BM, Washington D.C.,1991, dice: «Hay un interés cada vez mayor en la educación "tecnológica", con la cual se procura inculcar una comprensión más general de las matemáticas y las ciencias aplicadas en el contexto de la tecnología y la producción, en lugar de formar en destrezas ocupacionales específicas. Esos cursos no requieren efectuar inversiones en talleres y equipos costosos, como los que necesitan los programas de capacitación para reproducir el entorno laboral. Aunque los programas de educación tecnológica son demasiado nuevos para haberlos evaluado, quizás se justifique experimentar con ellos. Una limitación significativa para su aplicación generalizada sería la necesidad de formación en gran escala de maestros». No es obvio que esta sea una directiva, pero es cierto que la idea ha calado hondo en algunas propuestas de reformas educativas. 
1996). Estimamos que una adecuada alfabetización tecnológica, una formación general en tecnología, es mucho más importante que aquélla, dado que el impacto de las tecnologías sobre la vida diaria de la gente es mucho más directo que el de sus respectivas bases científicas (Buch, 2002).

Pero, ¿qué contiene el eslogan alfabetización tecnológica?, y ¿qué quiere decir la frase formación general en tecnología? Aquí el enfoque semántico cambia ligeramente: de definir la tecnología como «la manera de hacer las cosas que tiene cierta sociedad» ${ }^{5}$, pasamos a definirla como «aquello que hacen los tecnólogos», o, tal vez (lo que resulta más interesante) si queremos plantear la actividad tecnológica como una actitud humana general, "cómo actúan las personas ante un problema tecnológico» (Marpegán, 2001). En este cambio de definición reside gran parte de la diferencia entre CTS y ET.

Cabe preguntarse: ¿qué es lo que hacen los tecnólogos? Aquí es probable que no haya un acuerdo general entre ellos, pero, aún en contra de algunas de sus opiniones, creo que se puede hacer un resumen más o menos ajustado. Los tecnólogos aplican toda clase de instrumentos, técnicas y conocimientos a la resolución de un problema planteado por la realidad, cualquiera que sea su origen. Una postura que aquí rechazamos con el mayor énfasis es la famosa identificación de la tecnología con aplicar la ciencia para satisfacer las necesidades humanas $^{6}$. En cuanto al origen de los problemas, tradicionalmente hubo quien opinaba, con algo de ingenuidad, que los que la tecnología resuelve son planteados por las necesidades de la sociedad, pero ya Ortega, en uno de los textos liminares históricos de la reflexión sobre la tecnolo-

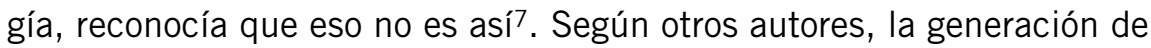

5 UNESCO (1983): «Technology Education as Part of General Education», en Science \& Technology Education Series, 4, París, define la tecnología en estos términos: «T. es el saber hacer y el proceso creativo que puede utilizar herramientas, recursos y sistemas para resolver problemas, para aumentar el control sobre el medio natural y el creado por los seres humanos, con objeto de mejorar la condición humana». Esta definición se parece a otras, pero resulta sumamente cuestionable la aplicabilidad de la última frase, "con el objeto de mejorar la condición humana».

6 Las primeras versiones del programa inglés SATIS (Science and Technology in Society) mencionan la siguiente definición: T. es «el proceso por medio del cual se hace posible la aplicación de la ciencia para satisfacer las necesidades humanas» (Citado en Alberto Maiztegui et al., en Revista Iberoamericana de Educación, núm. 28, OEI, 2002.

7 "La técnica es la reforma de la naturaleza, de esa naturaleza que nos hace necesitados y menesterosos, reforma en sentido tal que las necesidades queden a ser posible anuladas por dejar de ser problema su satisfacción», Ortega y Gasset (1939) "Meditación de la técnica», en Revista de Occidente, Madrid, 1977. 
esos problemas es muy variada, ya que muchas de las presuntas necesidades son generadas por la propia estructura que los pretende resolver, debido a la necesidad de expansión constante que es inherente a la economía capitalista (Galbraith, 1980; Le Monde, 2003). Pero la forma de trabajo del tecnólogo no se limita a la resolución de problemas, como pretenden algunos para los que este enfoque agota la ET. Por de pronto, la palabra problema es demasiado amplia. También una simple operación matemática es un problema en el sentido habitual que los docentes dan a esa palabra. Sin ajustar demasiado los términos, podríamos decir que la resolución de un problema tecnológico implica la creación o modificación de un objeto tecnológico, que puede ser un artefacto, un sistema tecnológico más amplio que un artefacto, o un proceso tecnológico (Buch, 1999). Por otra parte, ese concepto de resolución de problemas que, según algunos se limitaría casi a los de ingenio, a aquellos que se resuelven con el chispazo de la inspiración del inventor, está muy lejos de agotar la idea. En la resolución de un problema tecnológico entran muchas técnicas, entre las cuales podríamos citar (Buch, 1996) el análisis sistémico del problema, que involucra sus múltiples relaciones con los sistemas más amplios con los que se debe interactuar, y en los que la solución debe insertarse. Este sistema más amplio abarca aspectos tecnológicos, geográficos, ergonómicos, económicos, ecológicos y sociales ${ }^{8}$ de todo tipo, según la naturaleza del problema de que se trate. En este análisis pueden tener cabida la mayoría de los problemas abordados por CTS; el diseño de posibles soluciones conceptuales al problema, donde diseño es un concepto de gran amplitud, que abarca desde las actividades organizativas relacionadas con el objeto tecnológico que se quiere crear, hasta las más habituales de diseño gráfico; la construcción del sistema destinado a resolver el problema. La palabra construcción también se toma aquí en un sentido amplio, que incluye conceptos tales como organización o estructuración; el control de calidad, es decir, la verificación de que el sistema propuesto cumple de verdad con las condiciones requeridas para constituir una solución al problema planteado. En lo dicho anteriormente está implícito, pero vale la pena explicitarlo, que entre esas condiciones figuran en lugar destacado las económicas, que se analizaron antes. La importancia de éstas es tan relevante que se puede afirmar que si no las cumple, no existe solución, y, por lo tanto, no hay tecnología; todo este proceso es muy

8 Los aspectos sociales abarcan también los culturales. Un ejemplo de este tipo de condicionamientos es el rechazo de ciertos adelantos urbanos, como los servicios sanitarios, por las culturas que no aceptan que las necesidades se hagan dentro de la vivienda. 
iterativo, y durante el mismo todos los parámetros de la solución propuesta pueden variar.

Un dato significativo de la realidad es el hecho de que muchas de las actividades tecnológicas son esencialmente innovadoras. Su carácter dinámico es una de las características más destacadas de la cultura contemporánea, y ese rasgo también se ha introducido en las inquietudes pedagógicas, uno de cuyos objetivos debe ser el estímulo a la creatividad (López Cerezo y Valenti, 1999). De alguna forma, este concepto se ha incluido de manera destacada en la temática CTS que ahora habla, directamente, de CTS+I (OEI, 2002). Este dinamismo tiene, a su vez, aspectos positivos y negativos para cada destino individual y para el de las sociedades.

El concepto epistemológico central en todo objeto tecnológico es su finalismo, ya que es creado con una finalidad explícita. Así como es un grave error epistemológico decir que el ojo existe para ver, ya que se trata del resultado de un proceso evolutivo que carece de teleonomía, es evidente que un fotosensor artificial, que es su homólogo tecnológico, sólo existe porque alguien quiso construirlo teniendo su función como meta. El pensamiento finalista es una característica definitoria del proceso tecnológico, que lo diferencia de modo terminante del razonamiento científico al cual todo finalismo está vedado.

Otro concepto central de lo que con justicia se puede llamar pensamiento tecnológico es el de control. El control, en el sentido tecnológico de este término, implica la realimentación, que es una generalización no-lineal de la causalidad: la fuerza actuante en un proceso es controlada por el resultado de su acción. Esta realimentación, que está presente en todos los movimientos naturales de los organismos vivos, se hace explícita en el empleo de las herramientas, desde las más sencillas de uso manual hasta los sistemas tecnológicos más complejos.

El tercer elemento del pensamiento tecnológico es su carácter sintético. Allí donde el pensamiento científico es reduccionista y analítico, buscando averiguar las causas de los fenómenos, el pensamiento tecnológico parte de la función global del objeto tecnológico para descender luego al diseño de sus componentes.

Al mismo tiempo, el pensamiento tecnológico es sistémico, y sabe que cada objeto tecnológico individual, por abarcador que sea, es 
un subsistema de un sistema técnico ${ }^{9}$ coherente, que es característico de y caracteriza a cada época histórica.

En algunos enfoques (Doval y Gay, 1995) una de las etapas importantes de la ET es el análisis o la lectura de ciertos objetos tecnológicos, que no debería limitarse a su descripción y taxonomía. Esta lectura debería incluir sus procesos de generación y de producción, y evitar a toda costa sacarlos de su contexto. En concreto, no se debe perder de vista aquella parte del proceso de diseño que consiste en la búsqueda de compromisos entre los diversos factores -tecnológicos, económicos, ecológicos, mercadotécnicos, etc.- por los que se ve condicionado.

Por otra parte, tal descontextualización también puede abarcar otros aspectos del objeto, los que lo condicionaron desde un punto de vista social más amplio, que algunas veces son sutiles y difíciles de percibir, si no se los considera con una especial suspicacia. Así, se destacan los artefactos diseñados para diestros que son de complicado manejo para los zurdos, o aquellos basados en las costumbres occidentales que fracasan al pretenderse su traslado a otras culturas (Martín Gordillo y González Galbarte, 2002).

Como se ve, en toda esta descripción no se ha mencionado la palabra ciencia. Es que la ciencia, en esta concepción de la tecnología, es sólo uno más de los ingredientes o insumos que están disponibles para ser usados en la resolución del problema planteado. Esto no disminuye su importancia, pero la pone en una perspectiva muy diferente de la que implica la definición de tecnología como «ciencia aplicada».

La importancia de la ciencia para la tecnología contemporánea estriba en que la comprensión de los mecanismos íntimos de funciona-

9 Se nos debe perdonar aquí cierta terminología no muy coherente que se ha impuesto en la literatura. Si bien por lo general se estima que lo técnico tiene un nivel epistémico inferior a lo tecnológico, nosotros hemos llamado sistema tecnológico a los diversos subsistemas que, en su conjunto más amplio, forman el sistema técnico o tecnosfera que caracteriza una época o una civilización determinada. El sistema técnico es el conjunto de todas las tecnologías y de todos los objetos tecnológicos de esa civilización determinada. Los sistemas técnicos (paleolítico, neolítico, hidráulico, medieval, etc., así como chino, azteca, o mapuche) son conjuntos caracterizados por su coherencia interna, aunque cada uno tiene su propio nivel de complejidad. Cuando dos sistemas técnicos interactúan, generalmente se producen conflictos. El subdesarrollo es el conflicto generado en una sociedad que posee un sistema técnico menos complejo, cuando es expuesta al impacto de una sociedad de mayor complejidad a través de relaciones de dominio, como ha ocurrido en todas las sociedades dominadas por la civilización occidental en su expansión a partir del siglo xv. 
miento de la materia ha permitido optimizar sistemas tecnológicos, así como concebir objetos tecnológicos impensables sin ese conocimiento. Si la radio de galena pudo sustentarse en una observación casual, y la válvula electrónica -elemento fundamental de la tecnología electrónica de la primera mitad del siglo XX- se basó en conceptos científicos relativamente elementales como el efecto Edison, el transistor sólo es pensable a través de un conocimiento teórico avanzado de la estructura de bandas de los semiconductores empleados. En cambio, el proceso de fabricación de los circuitos integrados, que son la base de todos los sistemas electrónicos actuales, es un complejísimo desarrollo tecnológico frente al cual los propios principios físicos son sólo uno de los elementos que entran en juego. Lo mismo ocurre con numerosos productos farmacéuticos, que han evolucionado de compuestos químicos más o menos sencillos al diseño de moléculas y a los productos biotecnológicos actuales. El contraejemplo más clásico al concepto de la tecnología como ciencia aplicada es el de la máquina de vapor, que antecedió en mucho al descubrimiento de las leyes de la termodinámica, aunque éstas se usaran para perfeccionarla.

\section{4. ¿CÓMO SE VERÍA CTS DESDE ET?}

¿Cuál es, o podría ser, la relación entre CTS y ET? Como hemos visto, la ET, tal como entendemos ese término, analiza el pensamiento tecnológico y su manera de operar. La corriente CTS examina los modos en los que se generan los problemas y la construcción social de las soluciones, con especial énfasis en las consecuencias sociales y ambientales de las soluciones aplicadas. Sus contenidos son muy diferentes, pero sus relaciones deberían ser armónicas y permitir así una secuencia que se extendería a todo lo largo de la formación escolar primaria y secundaria, y completaría el ciclo de la alfabetización tecnológica necesaria para que un ciudadano pudiese formarse una opinión informada sobre un tema tecnológico cualquiera. En los primeros años, el énfasis estaría puesto en los contenidos técnicos, para ir creando poco a poco una comprensión cabal de los aspectos sistémicos, que se irían haciendo más complejos en la medida de la maduración de los alumnos.

Ya hemos mencionado que, en la primera fase del proceso tecnológico, se deben analizar los aspectos tecnológicos, geográficos, ergonómicos, económicos, ecológicos y sociales de todo tipo que se refieren al objeto tecnológico que hay que crear o modificar. Se deberían incluir también los aspectos éticos, sobre todo el de qué hacer cuando surgen 
conflictos entre los diversos aspectos mencionados. Aquí es donde el análisis tecnológico puro no es suficiente. La corriente CTS, al tratar de poner el objeto tecnológico en un contexto de máxima amplitud, y al no vacilar ante su enjuiciamiento ético y político, está en condiciones de añadir los aspectos sociales más profundos, las implicaciones éticas, las proyecciones políticas, el análisis de las intenciones que establecen el origen mismo del planteo del problema tecnológico: el porqué de su existencia.

Ahora bien, conforme existen diversas propuestas acerca de otros tantos modos de incluir los contenidos del enfoque CTS en el currículo educativo, es necesario analizar la manera en que ahora se abordarían desde los contenidos propios de la ET.

Se han intentado tres alternativas para la introducción de los contenidos CTS en el currículo (López Cerezo, 1998): CTS como asignatura, CTS como contenido de otras asignaturas, y CTS como pretexto para el estudio de la ciencia y la tecnología. Siempre se ha expresado la crítica de que todos estos enfoques relegan la tecnología a un estatus subalterno (irónicamente cts). Esta crítica tiene cierto cariz paradójico, ya que, además de la forma en que la ciencia afecta o determina la cosmovisión predominante, la manera más eficaz en que influye sobre la vida de la gente es a través de las tecnologías que la aplican. Abordar CTS desde la ET podría revertir esta situación, ya que la mayoría de los enfoques CTS, al poner otra vez demasiado énfasis en la ciencia, disimulan el proceso real del desarrollo tecnológico aunque oficialmente reniegue de la posición tecnología = ciencia aplicada.

Veamos entonces cuál de los tres enfoques mencionados sería el más apto para la combinación correcta entre la ET y la educación CTS. Aquí un análisis más profundo deberá tomar en cuenta las diferencias esenciales que existen entre las modalidades propias de los diferentes niveles del sistema educativo.

Por lo pronto, no creemos nada práctico el enfoque transversal de la tecnología en el currículo de las diferentes ciencias, ya que el profesor de una ciencia particular no está capacitado en general para enseñar tecnología, y estará tentado de considerar los temas tecnológicos que aborde en su clase como meras aplicaciones de su ciencia a un caso tecnológico determinado. 0 , peor aún, tomará las eventuales aplicaciones como pretextos para enseñar la ciencia de la misma manera que lo ha hecho siempre. En cambio, si, además de formar docentes en 
ET se pudiese lograr una verdadera capacitación tecnológica de los profesores de ciencias y convencerlos de que colaboren con aquellos, este método podría invertir el desinterés por la ciencia, y ser un aliciente para interesar a los alumnos en ella (Halbwachs, 1985).

Es decir, si bien se ha refutado muchas veces la caracterización de la tecnología como aplicación de la ciencia, y, por lo tanto, se acepta que no tiene sentido intentar deducir las aplicaciones tecnológicas de un hecho científico de ese hecho, en cambio la búsqueda de soluciones a problemas tecnológicos específicos ha sido un fuerte aliciente para la investigación científica, y puede serlo para la enseñanza de la ciencia. De ese modo, el orden habitualmente abordado por la educación quedaría del todo invertido, incluso en la educación superior: lejos de enseñar primero ciencia para luego aplicarla, se comenzaría por motivar la enseñanza de la ciencia en su necesidad para la resolución de los problemas tecnológicos (Maiztegui, 2002). El mismo criterio podría aplicarse también a la formación de los ingenieros (Bazzo, 2002; Buch, 1999b). Es muy probable que la motivación de la mayoría de los alumnos para estudiar ciencias se vería estimulada en gran medida por ese nuevo ordenamiento.

\section{CONCLUSIÓN}

De la comparación entre las ideas que inspiran a todos aquellos que proponen la profundización de los estudios relativos a la tecnología, sobre todo en la educación secundaria, surge que los dos enfoques principales, la corriente CTS que se aproxima a la problemática de la tecnología más desde las humanidades que desde las ciencias físicas y naturales o las técnicas, y la ET que llega a los aspectos ambientales y sociales de la tecnología proviniendo más de las técnicas, y, en ciertos enfoques, de su análisis antropológico, podrían combinarse en una síntesis superadora, con ventaja para ambos enfoques parciales y con plena ganancia para los alumnos, que de ese modo lograrían una visión más integral, a la vez que humanística y tecnológica de la tecnología en todos sus aspectos.

Para que esta síntesis pudiese hacerse efectiva con ganancia para los alumnos y para la formación cultural tecnológica de las generaciones futuras, debería darse un debate en profundidad sobre los aspectos estudiados en estas páginas, y otro referido a los contenidos curriculares y a su transposición didáctica. Además del hecho de que este 
enfoque combinado requiere esfuerzos aún mayores de capacitación de los profesores, los docentes tendrán que ser, en esta disciplina más que en las tradicionales, los guías en un mundo poco conocido que deberán descubrir junto a sus alumnos, dejando tal vez de lado, en ese camino, numerosos preconceptos y prejuicios.

Por desgracia, no puede excluirse que esa tarea resulte ser superior a las fuerzas disponibles, dado que, aún sin esta amplia síntesis, la formulación de la tecnología como área de estudio parece ofrecer grandes dificultades.

\section{BIBLIOGRAFÍA}

ACEVEDo DíAZ, José A.: «Educación tecnológica desde una perspectiva CTS», Sala de lectura CTSI, <http://www.campus-oei.org/salactsi/acevedo5.htm>. ga Editores.

ARgüELLES, A. (1999): La Educación tecnológica en el mundo, México, Norie-

BAzzo, W. A. (2002): «A pertinência de abordagens CTS na educação tecnológica», en Revista Iberoamericana de Educación, núm. 28, enero-abril, Madrid, OEI, <http://www.campus-oei.org/revista/rie28.htm>.

Buch, Tomás, «El Tecnoscopio transversal», inédito.

- (2002): Ciencia hoy, núm. 70, 2002, pp.18-27.

— (1999): Sistemas tecnológicos, Buenos Aires, Aique.

- (1999b): Ingeniería: discutamos desde las bases, Facultad de Ingeniería, Universidad del Comahue.

— (1996): El tecnoscopio, Buenos Aires, Aique.

ture, 7 (3).

Bunge, Mario (1996): «Technology as Applied Science», Technology and Cul-

CARES, Vladimiro (1999): «Ciencia y Tecnología: primera aproximación», Informe de la Facultad de Ingeniería, Universidad del Comahue, Neuquén.

DAGNINO, Renato: «Enfoques sobre a relação ciência, tecnologia e sociedade: neutralidade e determinismo", en Sala de lectura CTS+l, OEI, <http://www.campusoei.org/salactsi/rdagnino3.htm>.

DAGNINO, Renato y THOmAs, Hernán (2000): «Elements for an ExplicativeNormative Renovation of Latin American Policies of Innovation», en Espacios, vol. 21 (2), <http://www.revistaespacios.com/a00v21n02/14002102.html>. 
DoVAL, L. y GAY, A. (1995): «Tecnología: finalidad educativa y acercamiento didático», Buenos Aires, Prociencia - Conicet, <http://www.capacyt.edu.ar/pei3c.html>.

FOUREZ, G. et al. (1996): Alfabetización científica y tecnológica, Buenos Aires, Ediciones Colihue.

GALBRAITH, J. (1980): El nuevo estado industrial, Barcelona, Ariel.

GenNuso, Gustavo (2000): «Educación tecnológica en el nivel inicial, ¿una propuesta posible?», en Educación Tecnológica (Educación en los primeros años). Novedades Educativas.

GILBERT, J. K. (2002): «The Interface Between Science Education and Technology Education», en International Journal of Science Education, 14 (5) p. 563, Institute of Education, University of Reader, Reino Unido, Taylor \& Francis Group.

- (1995): «ET, una nueva asignatura en todo el mundo», en Revista de Investigación y Experiencias Didácticas, 1, vol. 13, Barcelona.

GonzÁlez García, M. I.; López Cerezo, J. A. y LujÁn LóPez, J. L. (1996): Ciencia, Tecnología y Sociedad, Madrid, Tecnos.

HALBWACHS, F. (1985): "La física del profesor entre la física del físico y la física del alumno», en Revista de Enseñanza de la Fisica, vol. núm 2, diciembre.

LE Monde (13/01/03): «Ces maladies inventées par les laboratoires», $<$ http://www.lemonde.fr/article/0,5987,3266-305195-,00.html>.

LÓPEZ CEREZO, José Antonio (1998): «CTS: el estado de la cuestión en Europa y los EE.UU.», en Revista Iberoamericana de Educación, núm. 18, septiembre-diciembre, Madrid, OEl, <http://www.campus-oei.org/oeivirt/rie18.htm>.

LÓPEZ Cerezo, J. A. y VAlentI, P. (1999): «Educación tecnológica en el siglo XXI», en Revista Polivalencia, núm. 8, octubre-noviembre, Fundación Politécnica/Universidad Politécnica de Valencia, España.

MalzTEGUI, A. et al. (2002): «Papel de la tecnología en la educación científica», en Revista Iberoamericana de Educación, núm 28, <www.campusoei.org/revista/rie28a05.htm>.

MARPEGÁn, Carlos (2001): «La Educación tecnológica en la lupa», en Novedades Educativas, núm. 128, agosto, pp. 34-37.

Martín Gordillo, M. y GonzÁlez Galbarte, J. C. (2002): «Reflexiones sobre la educación tecnológica desde el enfoque CTS», en Revista Iberoamericana de Educación, núm. 28, enero-abril, Madrid, OEl, <http://www.campus-oei.org/revista/rie28.htm>.

MoRIN, Edgar (2002): Ética y desarrollo, Banco Interamericano de Desarrollo, <http://www.iadb.org/etica/documentos/dc_mor_estam.htm>.

novich.

Mumford, Lewis (1970): The Myth of the Machine, Hartcourt, Brace \& Jova-

OEI (2002): Revista Iberoamericana de Ciencia, Tecnología, Sociedad e Innovación, <http://www.campus-oei.org/revistactsi/>. 
RoDRíGUEZ DE FRAGA, Abel (1996): «La incorporación de un área tecnológica a la educación general», en Propuesta Educativa, FLACSO, 7, núm. 15.

Buenos Aires, Aique.

- (1994): Educación tecnológica (se ofrece); espacio en el aula (se busca),

RODRIGUEZ DE FRAGA, Abel et al. (1999): «Educación tecnológica, pre-diseño curricular para la educación general básica», Dirección de Currícula, Secretaría de Educación, Gobierno de la Ciudad de Buenos Aires.

UNESCO (1983): «Technology Education as Part of General Education», en Science and Technology Education Document, Series, 4, París.

UNIVERSITÉ LIBRE DE BRUXELLES (2002): Sustainable Development, Virtual Library, <http://www.ulb.ac.be/ceese/meta/sustvl.html>.

VERILLION, Pierre (2000): «Instruments and Cognition: Piaget and Vigotsky Revisited», en International Journal of Science Education, vol. XXVI, Institute of Education, University of Reader, Reino Unido, Taylor \& Francis Group.

VILCHES, A. y FURIÓ, C. (1999): Ciencia, Tecnología, Sociedad: implicaciones en la educaicón científica para el siglo XXI», en Sala de lectura CTS+l, OEI, $<$ http://www.campus-oei.org/salactsi/ctseducacion.htm>.

WINNER, Langdon (1987): La ballena y el reactor, Madrid, Gedisa.

— (1979): Tecnología autónoma, Madrid, G. Gili. 